\title{
Local determination of ionospheric plasma convection from coherent scatter radar data using the SECS technique
}

\author{
O. Amm, ${ }^{1}$ A. Grocott, ${ }^{2}$ M. Lester, ${ }^{2}$ and T. K. Yeoman ${ }^{2}$ \\ Received 25 August 2009; accepted 25 September 2009; published 12 March 2010.
}

[1] A new technique for merging line-of-sight (LOS) data of the ionospheric plasma convection velocity, as obtained from coherent scatter radars, into a full velocity vector field on a sphere is presented. This technique is based on the expansion into Spherical Elementary Current Systems (SECS) which have been successfully applied to many other problems in ionosphere-magnetosphere physics. Despite their name mentioning currents for historical reasons, SECS can be used as basis functions for any continuously differentiable vector field on a sphere. In contrast to the traditional modeling of the radar data with spherical harmonics over the whole auroral zone, the new technique does not require any "a priori" model input but relies solely on the measured data, nor does it need any explicit boundary conditions to be specified. The new technique is designed to be applied locally to areas where sufficient radar backscatter exists. The analysis area that satisfies this condition may have any shape and is not limited to, e.g., spherical caps. A test with synthetic data shows that the method performs excellently (less than $5 \%$ relative error) if $25 \%$ or more of the optimal coverage of input data are actually available as backscatter data, with respect to the scale on which the results for the velocity vector field are desired to be obtained. Still if only $10 \%$ of the optimal coverage of input data are available, the technique performs fairly well with a relative error of $\sim 12 \%$. A second test with real LOS input data from the SuperDARN radars shows that on such a local area with sufficient backscatter, our new technique is able to reproduce mesoscale details of the LOS data significantly better than the current standard analysis based on the technique of Ruohoniemi and Baker (1998) which processes the radar data on the whole auroral zone. While the new technique is presented here for the application with LOS radar data, it can be applied for merging any kind of vector component data on a sphere to a vector field.

Citation: Amm, O., A. Grocott, M. Lester, and T. K. Yeoman (2010), Local determination of ionospheric plasma convection from coherent scatter radar data using the SECS technique, J. Geophys. Res., 115, A03304, doi:10.1029/2009JA014832.

\section{Introduction}

[2] The determination of ionospheric plasma convection maps is a key target for ionosphere-magnetosphere physics, since the convection and the electric field associated with it are crucial parameters for the determination of, e.g., ionospheric currents, field-aligned currents, and the energy deposition in the ionosphere. Presently, coherent scatter radars such as STARE [Greenwald et al., 1978] or the SuperDARN radar network [Greenwald et al., 1995; Chisham et al., 2007] are the only instruments able to determine such maps over a mesoscale region (of a few hundreds to

\footnotetext{
${ }^{1}$ Arctic Research Unit, Finnish Meteorological Institute, Helsinki, Finland.

${ }^{2}$ Department of Physics and Astronomy, University of Leicester, Leicester, UK.

Copyright 2010 by the American Geophysical Union. 0148-0227/10/2009JA014832\$09.00
}

thousands of kilometers) with a time resolution suitable to study single events (20 s for STARE, 1 min routinely with SuperDARN). In the future, with the advantages of modern electronic beam steering, SuperDARN can achieve higher time resolution. With the same technique, also the upcoming AMISR [e.g., Heinselman and Nicolls, 2008] and the EISCAT 3-D [Wannberg, 2008] incoherent scatter radars may be used for similar purposes. However, due to the much larger energy per volume required by incoherent scatter techniques, the results will be restricted to smaller areas or have less time resolution.

[3] The nature of coherent backscatter is spatially and temporally highly variable. That is, for each measured time step, only a certain percentage of the data points that would be observed in an optimal case are actually available. Furthermore, like with any radar, the coherent scatter radar only measures the component of the plasma convection velocity along the radar beam, called line-of-sight (LOS) velocity. In order to obtain a spatial map of the full convection vectors, therefore the available LOS velocities from two or more radars must be merged into a 2-D plasma 


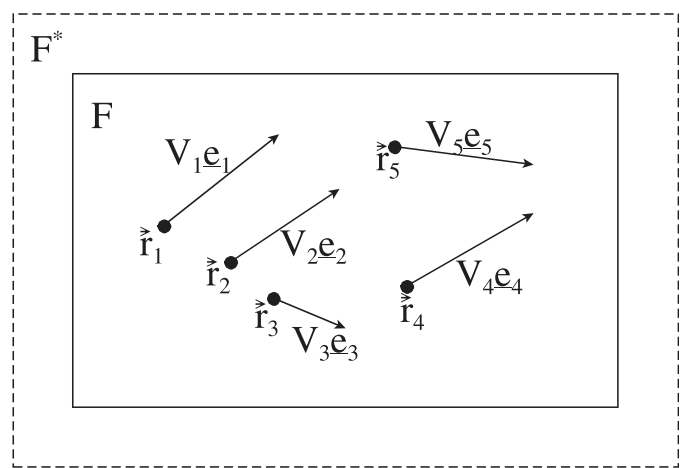

Figure 1. Sketch of the geometry of the vector component input data, and of the analysis region $\mathrm{F}$ as well as of the larger region $\mathrm{F}^{*}$, on which poles of elementary systems are placed.

convection velocity vector field. In most cases, also in the present paper, it is assumed that inductive effects can be neglected, and consequently the resulting 2-D velocity vector field is divergence-free.

[4] Plasma convection maps over the whole northern auroral zone are a standard output produced by the data of the SuperDARN coherent scatter radar network [Ruohoniemi and Baker, 1998]. However, in order to overcome data gaps either due to areas where none of the radars has a field of view, or due to lack of data because of missing backscatter, the technique to produce these maps (called "APL model" here) uses statistical "a priori" information taken from plasma convection models that are driven by parameters of the interplanetary magnetic field (IMF) and the solar wind. Further, the APL model is built on spherical harmonics, which means that missing information on one part of the analysis area will have an effect on all other parts of that area. For these two reasons, even in regions where good radar backscatter is available, it is not granted that the outcome of the APL model reflects the actual radar data in that region well. Particularly, there is a risk that smallerscale variations and anything in the data that is incompatible with the used statistical "a priori model" will be suppressed in the plasma convection maps produced by the APL model.

[5] In this paper, we present a new technique for merging plasma flow velocity vectors from LOS data locally in areas where sufficient backscatter from two or more coherent scatter radars is available, without the use of any "a priori" models. The new technique is based on Spherical Elementary Current Systems (SECS) which were introduced by Amm [1997], and since then have been used successfully in numerous tasks for ionosphere-magnetosphere physics, e.g., the determination of ionospheric equivalent currents from ground magnetometer data [Amm and Viljanen, 1999], the determination of induction effects in the ionosphere [Vanhamäki et al., 2006], or establishing a local version of the well known KRM technique [Kamide et al., 1981] that derives ionospheric electric fields and currents from ground magnetometer data, as shown by Vanhamäki and Amm [2007]. (Note that the notion "current systems" exists for historical reasons, but in fact the SECS can be used as basis functions for any kind of continuously differentiable vector field on a sphere.) The analysis region, i.e., the region with sufficient backscatter, may have any shape. The SECS basis functions are local, and thus no upper or lower wavelength that can be represented in the output results needs to be selected globally. In addition, no fixed boundary conditions need to be specified. The output plasma flow velocity vectors resulting from the technique are by definition exactly divergence-free.

[6] In section 2, we present the analytical basis of the new technique. In section 3, we test the technique using a synthetic data set, and by subsequently reducing the amount of available input data, we investigate how much backscatter is sufficient for the technique to operate with reasonable error margins. Finally, section 4 contains an application of the technique for a real SuperDARN data case. We compare the output of our new technique with that of the APL model on the same area, with the same input data.

[7] It should be noted that although the new technique is presented here in terms of merging LOS radar data to plasma convection velocity vectors, it is not restricted to this specific application. The mathematics presented in the following section can be applied to any type of vector component data on a sphere, and their merging into a vector field.

\section{Technique}

[8] In this section, we describe the analytical basis of the new technique, based on the SECS approach. For detailed description of the SECS approach itself, see Amm [1997] or Amm and Viljanen [1999].

[9] Figure 1 sketches the geometry of the input data, which consist of $n$ vector components $V_{i}$ in directions $\underline{e}_{i}, i=$ $1, \ldots, \mathrm{n}$, given at positions $\vec{r}_{i}$ on a spherical area F. In our radar data application, the unit vectors $\underline{e}_{i}$ refer to the LOS directions of the radar beams. The area $\mathrm{F}$ is the analysis area, in which the method provides the searched output of the technique, namely the vector field $\vec{V}$ on $\mathrm{F}$ such that $\operatorname{div}_{h} \vec{V}=$ 0 . Here and in the following, the subscript " $h$ " denotes the horizontal component of a vector or operator. For simplicity, in the sketch a rectangular area was chosen for F, but the shape of the analysis area can freely be chosen to best match the area of available input data.

[10] The way to solve for $\vec{V}$ with the SECS approach is to place $m$ divergence-free spherical elementary system poles to an area $\mathrm{F}^{*}$ with $F \subset F^{*}$, and then fit the scaling factors $I_{j, d f}, j=1, \ldots, m$ of these poles such that the field given by the sum of all SECS optimally fits the measured vector components. The elementary systems are placed with their poles on positions $\vec{r}_{j}^{\prime}$ on $\mathrm{F}^{*}$. Typically, the positions $\vec{r}_{j}^{\prime}$ are chosen as a regular grid, but the density of the elementary systems placed can also be adopted to reflect areas of denser or sparser input data availability if so desired.

[11] The first step of the solution is to solve for the scaling factors of the divergence-free elementary systems. For that, we solve the linear equation

$$
\underline{\underline{T}} \cdot \underline{I}=\underline{Z}
$$




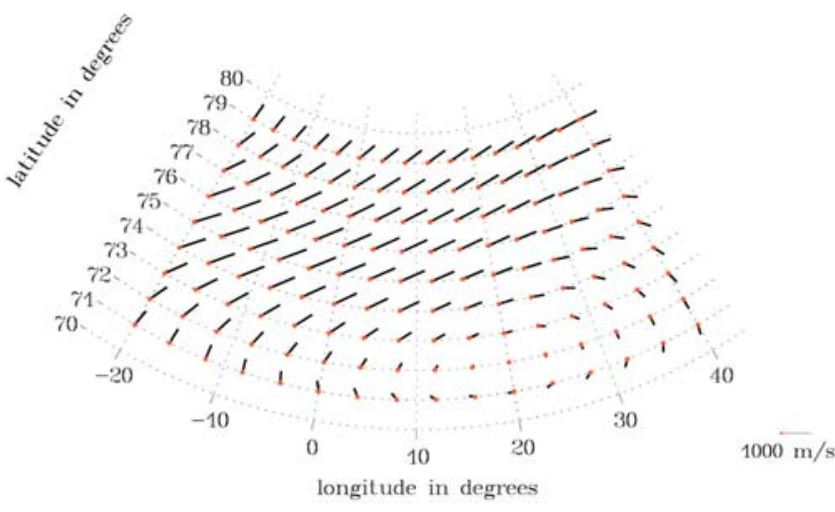

Figure 2. Synthetic model of a plasma flow velocity vector field, used to generate input data for testing the new technique with different backscatter probability levels.

with

$$
\underline{Z}=\left(\begin{array}{c}
V_{1} \\
V_{2} \\
\cdot \\
\cdot \\
\cdot \\
V_{n}
\end{array}\right)
$$

being the vector of observations, and

$$
\underline{I}=\left(\begin{array}{c}
I_{1, d f} \\
I_{2, d f} \\
\cdot \\
\cdot \\
\cdot \\
I_{m, d f}
\end{array}\right)
$$

being the vector that contains the searched scaling factors, and $\underline{T}=\left(T_{i j}\right)$ being the "transfer matrix" which gives the effect of a unit elementary system with pole at $\vec{r}_{j}^{\prime}$ in direction of $\underline{\mathrm{e}}_{\mathrm{i}}$ at point $\vec{r}_{i}$. Hence

$$
T_{i j}=\left(\frac{1}{4 \pi R_{I}} \cot \left(\vartheta^{*} / 2\right) \underline{e}_{\varphi}^{*}\right)^{t} \cdot \underline{e}_{i}
$$

where the expression inside the brackets is given in the spherical coordinate system of the elementary system with pole at $\vec{r}_{j}^{\prime}$, and the superscript " $\mathrm{t}$ " indicates that this expression needs to be transformed to the same coordinate system in which the $\underline{e}_{i}$ are given (i.e., usually the geographic or a geomagnetic coordinate system) before the dot product is taken. $\vartheta^{*}$ means the $\vartheta$ coordinate of $r_{i}$ in the SECS system with pole at $\vec{r}_{j}^{\prime}$, and $\underline{\mathrm{e}}_{\varphi}^{*}$ is the direction of the $\varphi$ unit vector of the same SECS system at $\vec{r}_{i}$. The transfer matrix $T$ is thus only dependent on the geometry, i.e., on the given location of the input vectors and on the chosen locations of the SECS poles, but it is independent of the actual flow velocity data. Using equations (2)-(4), equation (1) can be solved for $\mathbf{I}$ with the same singular value decomposition (SVD) inversion technique as described by Amm and Viljanen [1999, section 5].
[12] Once I (and therefore the scaling factors of each SECS system) is known, $\vec{V}$ follows immediately $\forall \vec{r}_{0} \subset F$ as

$$
\vec{V}\left(\vec{r}_{0}\right)=\sum_{j=1}^{m}\left(\frac{I_{j, d f}}{4 \pi R_{I}} \cot \left(\vartheta^{*} / 2\right) \underline{e}_{\varphi}^{*}\right)^{t}
$$

where again the expression in brackets is given in the spherical coordinate system of the elementary system with pole at $\vec{r}_{j}^{\prime}$, and the superscript " $\mathrm{t}$ " indicates the transformation to the global coordinate system before the sum is taken. $\vartheta^{*}$ means the $\vartheta$ coordinate of $r_{0}$ in the SECS system with pole at $\vec{r}_{j}^{\prime}$, and $\underline{\mathrm{e}}_{\varphi}^{*}$ is the direction of the $\varphi$ unit vector of the SECS system at $\vec{r}_{0}$. Equation (5) can be applied for any desired $\vec{r}_{0} \subset F$, and thus the searched vector field $V$ can be constructed.

\section{Test With Synthetic Data Sets}

[13] In this section, we test the performance of the new technique using a synthetic input data set of plasma flow velocity, as shown in Figure 2. In fact, this input data set has been constructed from SuperDARN measurements on 13 November 1996, 1900 UT, and it has been analyzed together with magnetometer data in the work of Amm et al. [1999]. However, in the context of this technique paper, we disregard the geophysical aspects and use the vector field shown in Figure 2 as a synthetic representation of a true plasma flow velocity distribution at some given time only.

[14] In order to test how well the new technique can reproduce the given synthetic input data set, and in order to test its sensitivity with respect to the availability of (or lack of) backscatter (and thus of input vector component data), we have performed the following virtual experiment: We define two "virtual radars," one of which measures only the north component, and the other only the east component of the original plasma flow velocity. (We are aware of the fact that in the real world, such a configuration would not be possible since lines of constant latitude are not straight on a sphere, but this is of no concern for this mathematical test.) The range gates of the "virtual radars" correspond just to the intervals between the vectors shown in Figure 2, i.e., if optimal backscatter would be received, the radar data would consist exactly of the north and east components of the vectors shown in Figure 2. Next, we define six probability levels $(100 \%, 75 \%, 50 \%, 25 \%, 10 \%$, and $5 \%)$ which define the probability for each vector component of the vectors shown in Figure 2 to be actually observed by the virtual radar. When generating virtual input data for each of the probability levels, a random process with the respective probability is used to determine for each vector component whether or not it is observed. Each probability level thus corresponds to different amounts of backscatter (on average, the percentage of backscatter received equals the probability level) and therefore of input data for the technique.

[15] With the available input vector component data at each probability level, we then use the new SECS-based local merging technique presented in section 2 to reconstruct the divergence-free plasma flow velocity field. The relative error $e$ between the recalculated field (denoted by the sub- 

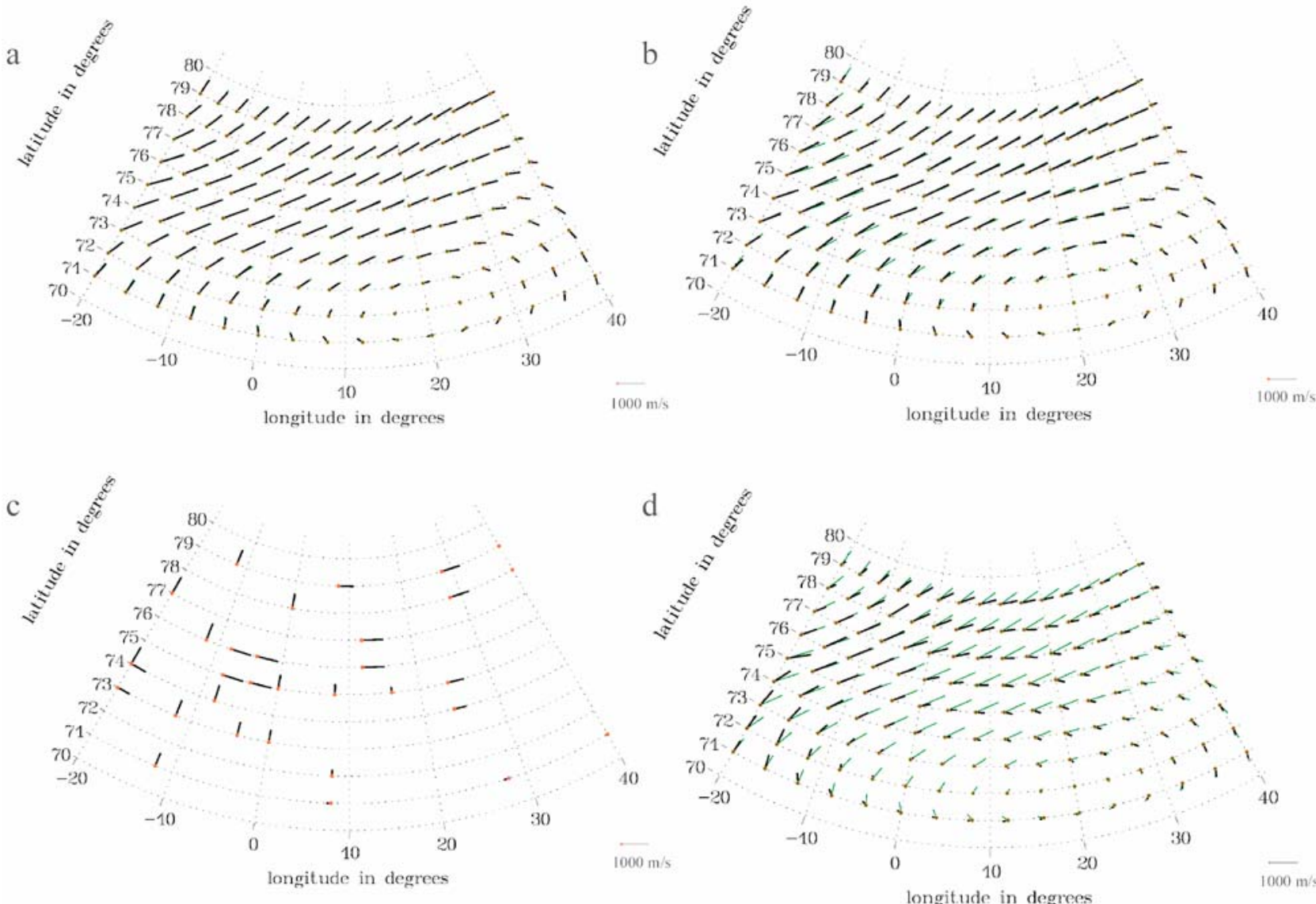

Figure 3. Reconstruction of the plasma flow velocity vector field from the synthetic model shown in Figure 2 using the new SECS-based radar merging technique, for different probability levels for the radar backscatter. (a) Output for $25 \%$ level; (b) output for $10 \%$ level; (c) input vector components for $10 \%$ level; (d) output for 5\% level.

script "calc") and the original field, i.e., the vectors shown in Figure 2 (denoted by the subscript "orig"), is defined as

$$
e=\frac{\int_{F}\left|\vec{v}_{\text {calc }}-\vec{v}_{\text {orig }}\right|}{\int_{F}\left|\vec{v}_{\text {orig }}\right|}
$$

[16] Figure 3 shows the output of the reconstruction with the new technique for the probability levels $25 \%, 10 \%$, and $5 \%$ (Figures 3a, 3b, and 3d, respectively). The reconstructed field is displayed by black vectors, and the original model field is displayed by green vectors, for comparison. The reason why we do not show the corresponding output for probability levels higher than $25 \%$ is clear from Figure $3 \mathrm{a}$ : Even at the $25 \%$ probability level, visually it is hard to observe any difference between the reconstructed and the original vector fields. However, the relative error $e$, given in Table 1, rises from $0.45 \%$ at $100 \%$ probability level to $4.41 \%$ at $25 \%$ probability level. The reason for $e \neq 0$ at $100 \%$ probability level is, in addition to numerical effects relating to the inversion, the fact that the amount of elementary system poles that we use for the SECS expansion is finite. At $10 \%$ probability level (Figure $3 \mathrm{~b}$ ), deviations between the reconstructed and the original vector fields start to become visual, particularly in the western part of the analysis area. Still, also at this low probability level, the structure of the original field is fully reconstructed, and the deviations are moderate with a relative error of $e=12.26 \%$. Figure $3 \mathrm{c}$ shows the vector component input data for this level, demonstrating the sparseness of the input data with which the technique still obtains a very reasonable reconstruction result. Finally, at 5\% probability level, the reconstructed vector field has clearly deteriorated (Figure 3d), and the relative error rises to $e=$ $49.45 \%$. However, even at this very low probability level, the result is not random but the basic features of the original vector field, i.e., the change in vorticity from northwest to southeast, the vortex at the southern flank, and the general decrease of the field's magnitude from west to east, are still recognizable.

\section{Test With a Real SuperDARN LOS Data Set and Local Comparison With APL Model Results}

[17] The second test of our technique is based on real SuperDARN LOS plasma velocity data, measured on 5 January 
Table 1. Relative Error $e$ of the Reconstructed Plasma Flow Velocity Vector Field Compared to the Original Synthetic Model Field, for Different Probability Levels for the Radar Backscatter ${ }^{a}$

\begin{tabular}{cc}
\hline Probability Level (\%) & Relative Error $e(\%)$ \\
\hline 100 & 0.45 \\
75 & 0.91 \\
50 & 2.20 \\
25 & 4.41 \\
10 & 12.26 \\
5 & 49.45 \\
\hline
\end{tabular}

${ }^{\mathrm{a}}$ Relative error $e$ is as defined in equation (6).

2000 at 2007 UT. In addition to the testing of the technique with real data, we locally also compare the results with those of the APL model, based on the same input data. We concentrate here on the field of view of the two CUTLASS radars which are part of the SuperDARN network and situated in Hankasalmi (Finland) and Pykkvibaer (Iceland). The location of the radars, their fields of view, and the observed LOS velocities are shown in Figure 4a. In the color scale, negative values and red/orange color denote LOS velocity components away from the radar, while positive values and blue/green colors denote LOS velocity components toward the radar. The data are presented in altitude adjusted corrected geomagnetic coordinates (AACGM [e.g., Gustafsson et al., 1992]). The interval presented is characterized by a very good level of available backscatter overall in the SuperDARN radars, and thus also for the CUTLASS radars. Still, some regions where no backscatter is received exist especially at the flanks of the field of view in the far range gates in the Pykkvibaer radar (Figure 4a, left), and at some medium distance and far range gates in the Hankasalmi radar (Figure 4a, right). Again, we do not discuss any geophysical aspects of the data set in this technique paper, but merely use it as an input to our technique.

[18] Figure 4b shows the output of the APL model on the northern auroral zone. In Figure $4 b$, magnetic noon is on the top, and magnetic midnight is on the bottom. For better orientation, also the two CUTLASS radars and their field of view are indicated, where "E" denotes to Pykkvibaer radar, and "F" denotes the Hankasalmi radar. The origins of the
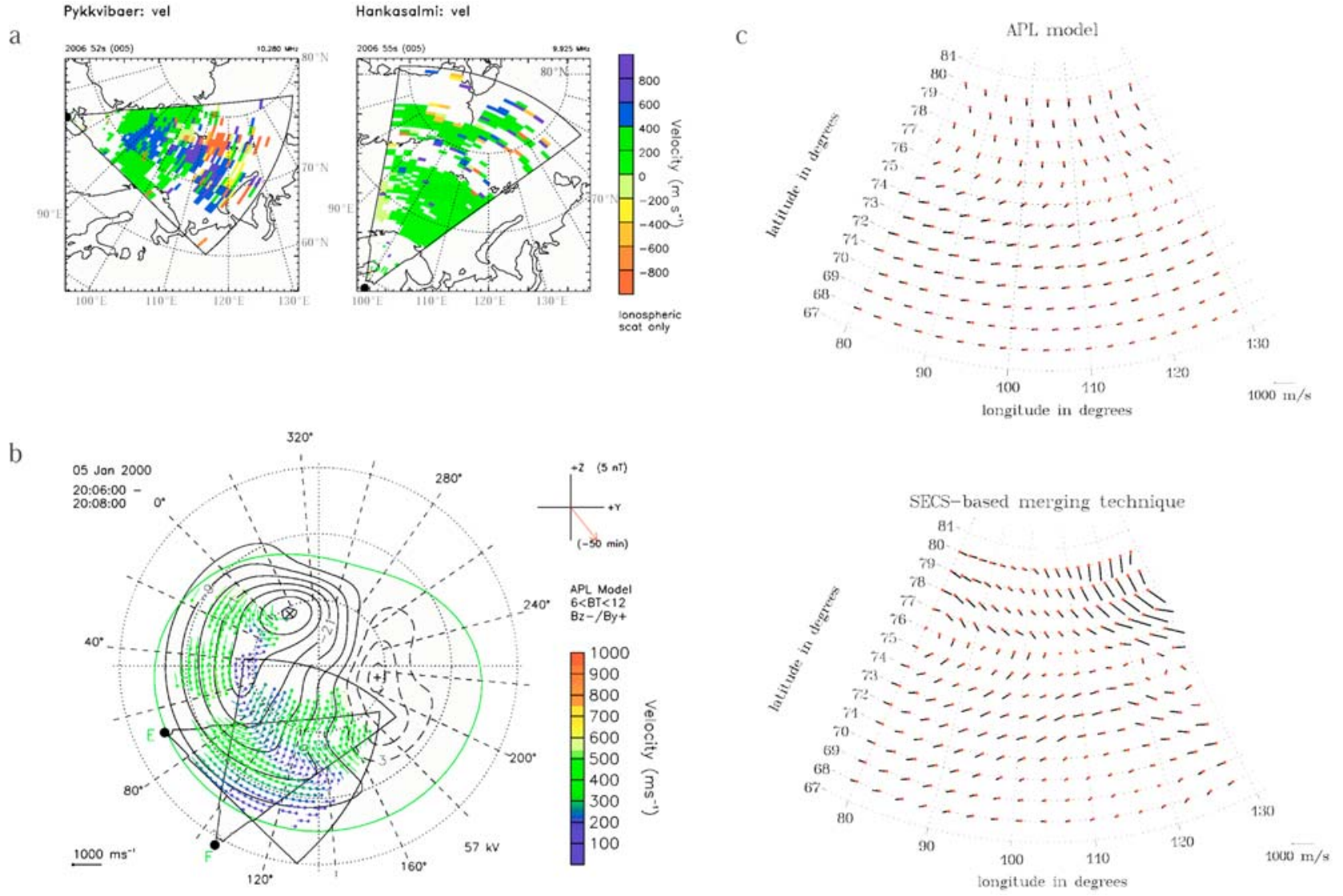

Figure 4. Testing of the new SECS-based radar merging technique with real SuperDARN data and local comparison with the results of the APL model. (a) Original LOS data from the two CUTLASS radars on 5 January 2000 at 2007 UT. (b) Resulting electric potential (isocontour lines) and plasma flow velocity (vectors) on the northern auroral zone from the APL model. The positions and field of view of the CUTLASS radars are marked. Magnetic noon is on the top of the plot, and the evening side is on the left. (c) Local comparison of results for the plasma flow velocity in the area of the CUTLASS radars' field of view: (top) APL model and (bottom) new SECS-based radar merging technique. 
vectors indicate positions where at least one of the SuperDARN radar obtained ionospheric backscatter. However, the direction of the vectors at these positions always point along the APL model solution for the plasma flow velocity (not along the original LOS measurement direction). In addition, also isocontours of the electric potential that describes the electric field $\vec{E}=-\vec{V} \times \vec{B}$ resulting from the APL model of $\vec{V}$ (where $\vec{B}$ denotes the Earth's main magnetic field) are plotted. As can be seen from Figure $4 b$, on the magnetic evening (i.e., on the plot at left) side of the auroral zone, the radars generally obtain a good amount of backscatter, while on most of the magnetic morning (i.e., on the plot at right) side, backscatter is sparse or fully absent. In these regions, the APL model output is dominated by the "a priori" information given to the model, which are based on parameters of the IMF also stated in Figure 4b. However, we emphasize that the nature of spherical harmonic basis functions, on which the APL model is built, is such that there cannot be a clear separation into areas that are influenced by the "a priori" information and others that would not be influenced by them. Moreover, the sparse data availability in some areas globally limits the maximum possible degree of the spherical harmonic expansion, and by that its spatial resolution.

[19] In Figure 4c, we directly compare the output of the APL model and that of our new SECS-based merging technique, in the area of the CUTLASS radar field of view. Looking only at the most basic features of the plasma flow velocity pattern, there is good correspondence between the results of the two techniques: Mostly westward flow is seen in the southern part of the analysis area equatorward of $73^{\circ}$ lat, while poleward of that the plasma flow splits into a clockwise vortical structure on the western side, and an anticlockwise vortical structure on the eastern side. However, when looking more closely, it becomes clear that the result of the new SECS-based technique preserves much more of the mesoscale detailed structures that are contained in the measured LOS velocity data (Figure 4a), and it also preserves a much larger variability of the amplitude of the plasma flow velocity, again in accordance with the original LOS data. For example, the intensification of the eastward flow in the northeastern edge of the analysis area and the strong zonal shear flow equatorward of it, as they are clearly seen in the LOS data of the Pykkvibaer radar, are both well represented in the results of the new SECS-based technique, but hardly visible in the results of the APL model.

\section{Summary and Discussion}

[20] In this paper, we have devised a new technique based on SECS for merging vector component data on a sphere into a vector field, and presented it for the specific case of coherent scatter radar LOS data of the ionospheric plasma flow velocity. Most importantly, unlike the traditional APL model that is regularly used with SuperDARN data on the whole northern auroral zone, the new technique does not require any "a priori" modeling nor other specific assumptions such as boundary conditions for the scalar potential that describes the plasma flow velocity vector field. On the other hand, the new technique must only be applied on areas where sufficient backscatter from two or more radars is present. These areas may have any shape, and are not restricted to, e.g., spherical caps, as is the case for techniques based on local spherical harmonics such as spherical cap harmonic analysis (SCHA [Haines, 1985]). Some inherent problems of SCHA related to the boundary conditions assumed in their construction were pointed out by Thébault et al. [2006].

[21] We have tested the new technique for one synthetic model case and for one real data case. The test with the synthetic model showed that the technique is able to produce excellent reconstructions of the original vector field with relative errors less than $\sim 5 \%$ when more than $\sim 25 \%$ of the original input vector component data are available as backscatter measurements. Even if only $\sim 10 \%$ of the original input vector component data are available, the reconstruction is still fairly good with a relative error of $\sim 12 \%$, and all structural features of the vector field being fully contained in the reconstruction. This is particularly reconfirming for the performance of our new technique because the synthetic model shown in Figure 2 is not an easy case for a local reconstruction, since it contains opposite vorticities, a wide dynamic range of vector magnitudes, and a vortex that is cut at the equatorward boundary of the analysis area. The above stated numbers of available backscatter can be used as guidelines for what amount of input data is sufficient for the application of the technique, with respect to the scale on which the results for the velocity vector field are desired to be obtained. (In our example, this scale is $\sim 100 \mathrm{~km}$.)

[22] The test of our new SECS-based merging technique with real SuperDARN LOS data, and the local comparison with the output of the APL model, showed that while the most essential features of the plasma flow velocity vector field agree, the results of the new technique contain much more mesoscale features of the vector field, and variability of its magnitude. Those features, which are clearly present in the input LOS data, are largely suppressed in the APL model results, presumably due to the global spherical harmonic basis functions used, and due to the influence of the "a priori" modeling. Therefore, in situations when mesoscale details of the radar data are of importance, or when the radar data is to be combined with other mesoscale data sets, in regions where sufficient backscatter exists it is recommendable to analyze the radar data with the SECS-based technique presented in this paper.

[23] For the present paper, we have restricted our discussion to the application of the new technique with a plasma flow velocity field that is desired to be divergencefree. Therefore, only divergence-free elementary system terms appear in equations (3)-(5). If for some other application, the output field is desired to be curl-free, then only curl-free elementary system terms would need to be used instead. If the output field should be of general kind, the matrix $T$ and the vector of scaling factors $\underline{I}$ need to be extended to contain both curl-free and divergence-free elementary system terms, and equation (5) would then contain the sum of both of these terms.

[24] Acknowledgments. The work of O.A. was supported through project 115947 of the Academy of Finland. A.G., M.L., and T.K.Y. were supported during this study by STFC grant PP/E000983/1. SuperDARN operations at the University of Leicester are supported by STFC grant $\mathrm{PP} / \mathrm{E} 007929 / 1$. 
[25] Wolfgang Baumjohann thanks Peter Dyson and Karl-Heinz Glassmeier for their assistance in evaluating this paper.

\section{References}

Amm, O. (1997), Ionospheric elementary current systems in spherical coordinates and their application, J. Geomagn. Geoelectr., 49, 947.

Amm, O., and A. Viljanen (1999), Ionospheric disturbance magnetic field continuation from the ground to the ionosphere using spherical elementary current systems, Earth Planets Space, 51, 431.

Amm, O., M. J. Engebretson, R. A. Greenwald, H. Lühr, and T. Moretto (1999), Direct determination of IMF $B_{Y}$-related cusp current systems, using SuperDARN radar and multiple ground magnetometer data - a link to theory on cusp current origin, J. Geophys. Res., 104, 17,187.

Chisham, G., et al. (2007), A decade of the Super Dual Auroral Radar Network (SuperDARN): Scientific achievements, new techniques and future directions, Surv. Geophys., 28, 33-109, doi:10.1007/s10712-007-9017-8.

Greenwald, R. A., W. Weiss, E. Nielsen, and N. R. Thomson (1978), STARE: A new radar backscatter experiment in northern Scandinavia, Radio Sci., 13, 1021

Greenwald, R. A., et al. (1995), DARN/SuperDARN: A global view of the dynamics of high-latitude convection, Space Sci. Rev., 71, 761.

Gustafsson, G., N. E. Papitashvili, and V. O. Papitashvili (1992), A revised corrected geomagnetic coordinate system for Epochs 1985 and 1990 J. Atmos. Terr. Phys., 54, 1609-1631.

Haines, G. V. (1985), Spherical cap harmonic analysis, J. Geophys. Res., 90, 2583.
Heinselman, C. J., and M. J. Nicolls (2008), A Bayesian approach to electric field and $E$ region neutral wind estimation with the Poker Flat Advanced Modular Incoherent Scatter Radar, Radio Sci., 43, RS5013, doi:10.1029/ 2007RS003805.

Kamide, Y., A. D. Richmond, and S. Matsushita (1981), Estimation of ionospheric electric fields, ionospheric currents and field-aligned currents from ground magnetic records, J. Geophys. Res., 86, 801.

Ruohoniemi, J. M., and K. B. Baker (1998), Large-scale imaging of highlatitude convection with super Dual Auroral Radar Network HF radar observations, J. Geophys. Res., 103, 20,797.

Thébault, E., J. J. Schott, and M. Mandea (2006), Revised spherical cap harmonic analysis (R-SCHA): Validation and properties, J. Geophys. Res., 111, B01102, doi:10.1029/2005JB003836.

Vanhamäki, H., and O. Amm (2007), A new method to estimate ionospheric electric fields and currents using data from a local ground magnetometer network, Ann. Geophys., 25, 1141.

Vanhamäki, H., O. Amm, and A. Viljanen (2006), New method for solving inductive electric fields in the ionosphere, Ann. Geophys., 24, 2573.

Wannberg, G. (2008), EISCAT-3D: The third generation European Incoherent Scatter radar system, in Proceedings of Radio Science and Communications and Mathematical Modelling of Wave Phenomena, edited by S. Nordebo and B. Nilsson, pp. 140-144, Springer, New York.

O. Amm, Arctic Research Unit, Finnish Meteorological Institute, PO Box 503, FIN-00101 Helsinki, Finland. (olaf.amm@fmi.fi)

A. Grocott, M. Lester, and T. K. Yeoman, Department of Physics and Astronomy, University of Leicester, Leicester LE1 7RH, UK. 